\title{
Customised fixed appliance systems and treatment duration
}

\author{
Abstracted from \\ Penning EW, Peerlings RHJ, Govers JDM, Rischen RJ, Zinad K, Bronkhorst EM, Breuning KH, Kuijpers-Jagtman AM. \\ Orthodontics with Customized versus Noncustomized Appliances: A Randomized Controlled Clinical Trial. J Dent Res 2017; 96: 1498-1504. \\ Address for correspondence: AM Kuijpers-Jagtman, Department of Orthodontics and Craniofacial Biology, Radboudumc, 309 Dentistry, \\ PO Box 9101, 6500 HB, Nijmegen, the Netherlands. E-mail: AnneMarie.Kuijpers-Jagtman@radboudumc.nl
}

\section{Question: Is there a difference in treatment duration between a customised fixed appliance system and a noncustomised system?}

\section{Design Randomised controlled trial.}

Intervention Patients aged 12-30 years requiring fixed orthodontic treatment were eligible and were randomly allocated to treatment with the Insignia customised orthodontic system or the Damon Q noncustomised orthodontic system.

Outcome measure The primary outcome measure was treatment duration. Secondary outcomes were quality of treatment result; the degree of improvement graded using the Peer Assessment Rating (PAR) score; number of visits from the first visit after bonding to debonding; number of loose brackets; time required for treatment planning; and number of complaints.

Results One hundred and eighty patients entered the trial. Four were lost to follow-up and two did not complete treatment, so 174 were analysed. Treatment duration was $1.29 \pm 0.35$ years in the customised group and $1.24 \pm 0.37$ years in the noncustomised group. The PAR did not differ significantly between groups. However, the orthodontist had a significant effect on treatment duration, quality of treatment outcome and number of visits $(P<0.05)$. Compared to the noncustomised group, the customised group had more loose brackets, a longer planning time and more complaints.

Conclusions The customised orthodontic system was not associated with significantly reduced treatment duration, and treatment quality was comparable between the two systems.

\section{Commentary}

This trial addresses an important and relatively underexplored clinical question: 'Is there a difference in treatment duration between a customised fixed appliance system (Insignia) and a noncustomised system (Damon Q)?' Secondary outcomes included quality of treatment result, planning time and number of loose brackets, visits and complaints. The outcomes considered are of value to both orthodontists and patients. This RCT adds a higher level of evidence to the existing knowledge around the efficiency of customised appliances, which has otherwise consisted mostly of expert opinion, case reports and a retrospective study, which suggested that orthodontic treatment with Insignia is shorter. ${ }^{1,2}$

The study addressed a clearly focused research question and overall the quality of the methodology seems robust: randomisation and allocation concealment were well done; the tool used to assess the quality of treatment results, the Peer Assessment Rating (PAR) score is validated, reproducible and has been widely used; ${ }^{3}$ the study included the masking of the outcome assessor, often the only person who can be masked in intervention studies such as this; loss to follow up was small and intention to treat analysis was used.

Duration of treatment did not differ but the planning time in the customised group was longer. No details are given about how the planning time was measured but the average was 89 minutes for the customised group and 12 minutes for the non-customised. It is possible that this extra time might have been compensated for at the chairside by a reduced bonding time, but this information was not reported.

Although malocclusions were comparable between groups at baseline, only mild malocclusions were included. This will affect the generalisability of the findings to more severe malocclusions. Further investigation is needed to assess whether the findings would be similar in more complicated cases.

For the time being, based on this clinical trial there is insufficient evidence to encourage the use of customised appliances over non-customised.

Practice point

- No significant difference was found in treatment duration and quality of outcomes between the two groups.

- Planning time and number of loose brackets and complaints were higher in the customised group.

\footnotetext{
Margarita Papakostopoulou ${ }^{a}$ and Dominic Hurst ${ }^{b}$

a Postgraduate trainee in Orthodontics, Charles University, Prague ${ }^{b}$ Centre for Dental Public Health and Primary Care, Barts and The London School of Medicine and Dentistry, Queen Mary University of London, London, UK and Department of Primary Care Health Sciences, University of Oxford, Oxford, UK

1. Aldrees AM. Do customized orthodontic appliances and vibration devices provide more efficient treatment than conventional methods? Korean / Orthod 2016; 46: 180-185.

2. Weber DJ 2nd, Koroluk LD, Phillips C, Nguyen T, Proffit WR. Clinical effectiveness and efficiency of customized vs. conventional preadjusted bracket systems. / Clin Orthod 2013; 47: 261-266.

3. Richmond S, Shaw WC, O'Brien KD, et al. The development of the PAR Index (Peer Assessment Rating): reliability and validity. Eur J Orthod 1992; 14: 125-139.
}

Evidence-Based Dentistry (2018) 19, 50. doi:10.1038/sj.ebd.6401306 